\title{
Ganancia educativa en la educación media superior
}

\section{Learning gains in higher middle education}

\author{
Rodolfo Tuirán, José Luis Gaviria, Rubén Lugo, Daniel Hernández \\ y Miriam Benítez \\ El Colegio de México, México, Universidad Complutense de Madrid, España \\ Centro Nacional de Evaluación para la Educación Superior, México, \\ Tecnológico de Monterrey, México \\ Emerging Markets Political Risk Analysis, México
}

Resumen

Se presentan los resultados del primer estudio a nivel nacional en México de la ganancia educativa de los alumnos de tres generaciones, quienes concluyeron su educación media superior a partir de 2013. Se analizan datos de pruebas estandarizadas ENLACE de tercero de secundaria y de ENLACE/PLANEA de tercer grado de bachillerato, a través de reactivos ancla que midieran constructos semejantes en dos momentos en el tiempo para los mismos estudiantes. La comparación de medias de habilidad en los dos momentos de evaluación permite identificar que hay avance en el rendimiento de los alumnos al concluir el bachillerato, independientemente de la institución, algunas características individuales y el lugar donde se ubique la escuela. Se presentan análisis bivariados y multivariados, identificando diferencias en la ganancia educativa de acuerdo con el subsistema de educación media superior en que se estudió, turno al que asistió, tipo de secundaria del que provinieron y sexo de los estudiantes, entre otras variables.

Palabras clave: Ganancia educativa en México, educación media superior, diferencias en logros de aprendizaje entre grupos de población.

\section{Abstract}

We present the results of the first national study on learning gains in Mexico related to the experiences of three generations of students who complete their higher middle education, as of 2013 and until 2015. We used data from standardized third grade ENLACE tests from secondary schools and from third grade higher middle education ENLACE/PLANEA exams. The instruments contain anchor questions measuring similar constructs at two moments in time for the same students. The comparison of students's learning achievement in these two evaluation moments, allows us to identify that there was progress in their performance after having completed their higher middle education, regardless of the institution, some individual characteristics and the place where the school is located. Bivariate and multivariate analyzes are presented, identifying differences in the students' learning gains, according to the subsystem of higher middle education in which they studied, the school shift attended, the type of secondary school from which they came from, and their sex, among other variables.

Keywords: Learning gains in Mexico, higher middle education, differences in learning achievement among population groups. 


\section{INTRODUCCIÓN}

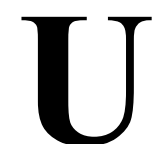

no de los principales desafíos de la educación en los diferentes niveles en México consiste en lograr una efectiva mejora de los resultados de los aprendizajes de niñas, niños y jóvenes. Para llamar la atención acerca de la profundidad del problema educativo en México, un conocido investigador afirmó, por ejemplo, que en el nivel medio superior "los jóvenes que terminan el bachillerato tienen, cuando más, nivel de tercero de secundaria". ${ }^{1}$ Uno puede preguntarse de inmediato si efectivamente esta afirmación tiene sustento empírico suficiente.

Para atender este tipo de preocupaciones, surgió la noción de ganancia educativa, la cual está relacionada directamente al concepto de valor añadido y eficacia escolar (Cervini y Dari, 2008), y alude al progreso en los aprendizajes durante periodos de tiempo determinados (generalmente niveles escolares sucesivos) debido a la acción escolar (Chudowsky et al., 2010; Kane, 2017; Simkrovic, 2017; Amrein-Beardsley et al., 2013). ${ }^{2}$

Los modelos de ganancia educativa se basan en la medición de resultados de una misma persona en el tiempo (Yeow Meng Thum, 2009), lo que en parte implica contar con una medida de aislamiento de los componentes que se refieren directamente a los factores educativos (que influyen en los resultados escolares), separándolos de otros factores intervinientes, inherentes al sujeto o que se refieren a aspectos socioeconómicos presentes en cada estudiante (Miranda, 2008).

De acuerdo con Gaviria (2006), la ganancia educativa de los alumnos se puede valorar a través de expresiones empíricas de la distancia que existe entre el nivel actual de conocimientos y un nivel previo. Para lograr la medición de valor agregado se pueden utilizar métodos como el escalamiento o equiparación vertical, que se emplea para vincular puntuaciones de instrumentos que evalúan el mismo constructo, pero que difieren en el nivel de dificultad (y están destinados a distinto grado o año escolar). Además,

1 La frase citada es atribuida a Roberto Rodríguez. Véase Revista Proceso, 13 de octubre de 2017.

2 Las medidas de valor añadido tienen dos elementos clave: por un lado, el progreso en el aprendizaje de los alumnos en periodos sucesivos; y por el otro, el rendimiento de los alumnos de forma individual (Gaviria y Castro, 2010). Realmente el valor añadido tiene como componentes, por una parte la ganancia, es decir, la diferencia entre el rendimiento inicial y el rendimiento final, y sobre todo, qué parte de esa ganancia puede atribuirse en exclusiva a la acción de la escuela. Por eso es importante controlar muchas variables contextuales que pueden explicar gran parte de las diferencias 
se siguen diseños de aplicación de los instrumentos de forma longitudinal y de modelos estadísticos para identificar los indicadores que dan información de los resultados atribuibles al sistema de enseñanza.

Gaviria y Castro (2010) señalan que los modelos de ganancia educativa tienen las siguientes características comunes:

- Utilizan como variables dependientes las respuestas de los estudiantes en diferentes instrumentos de evaluación de logro académico.

- Suelen incluir características contextuales de los alumnos y las escuelas como covariables.

- Son modelos cuantitativos que incluyen dos o más medidas de rendimiento.

- La relación funcional del crecimiento depende del número de mediciones.

- Suelen tratarse estadísticamente a través de modelos mixtos de crecimiento. ${ }^{3}$

Para realizar el escalamiento, es necesario vincular instrumentos que evalúen el mismo constructo en diferentes niveles. Además, para realizar inferencias validas a partir de un escalamiento o equiparación vertical, una condición que se debe asegurar es la adecuación de las escalas entre las medidas de rendimiento académico de los niveles que se están comparando.

Es indiscutible que en México se vienen realizando importantes esfuerzos para incrementar la cobertura, mejorar la infraestructura, diseñar nuevos currículos y formar a los docentes, entre otros aspectos. Sin embargo, aún persisten problemas de calidad de la educación que afectan en mayor medida a los jóvenes en situación de vulnerabilidad. En este sentido, es preciso asignar prioridad al desarrollo de políticas educativas que tengan como propósito potenciar el papel de la acción escolar en cada nivel educativo.

Existe evidencia que muestra que la acción escolar que se despliega en cada nivel educativo puede marcar la diferencia en el desempeño de los estudiantes. El propósito esencial de este artículo es aportar evidencia acerca de la influencia de la escuela en el desempeño de los alumnos y me-

\footnotetext{
3 De acuerdo con Gaviria y Castro (2005) los modelos mixtos de crecimiento o también conocidos como modelos lineales jerárquicos, modelos anidados, modelos multinivel, etc., son, en esencia, ampliaciones de los modelos de regresión lineal clásicos; ampliaciones mediante las cuales se elaboran varios modelos de regresión para cada nivel de análisis. Con ello, los modelos del primer nivel están relacionados por un modelo de segundo nivel en el que los coeficientes de regresión del nivel 1 se regresan en un segundo nivel de variables explicativas, y así sucesivamente para los diferentes niveles (Snijders y Bosker, 1999; Kreft y Leeuw, 1998).
} 
dir la ganancia educativa que significa cursar la Educación Media Superior (EMS) en el país.

\section{MÉTOdos y FUenTES de DATOS}

Una de las áreas que se consideraron en el desarrollo de ENLACE-MS (Evaluación Nacional del Logro Académico en Centros Escolares de Educación Media Superior) consistió en proveer información que permitiera conocer la ganancia que representa cursar la educación media superior (EMS) en México. ENLACE-MS se aplicaba a los estudiantes del tercer grado de bachillerato. Para atender este propósito, se buscó desarrollar y alinear las puntuaciones de las pruebas ENLACE-MS con el instrumento aplicado a los alumnos que terminaban su secundaria tres años antes (ENLACE-tercero de Secundaria).

El ENLACE MS se abocó a la medición del nivel de dominio de los alumnos del último ciclo de bachillerato en Comunicación y Matemáticas, las cuales se consideran estructuradoras fundamentales de los aprendizajes.

En la medición del desempeño se utiliza, en el caso de Matemáticas, un modelo que combina distintos contenidos temáticos; y en el caso de Comunicación se emplean tipos de texto que sirven de contexto para plantear situaciones al alumno que le exigen la puesta en práctica de grupos de procesos cognitivos y tareas de menor a mayor complejidad. Esto permite emitir los resultados de la prueba en cuatro diferentes niveles de dominio que sirven para caracterizar las fortalezas y debilidades de los sustentantes que participan anualmente en la aplicación.

Además de ofrecer una prueba diagnóstica para los alumnos del último ciclo de bachillerato, ENLACE-MS se propuso para conocer la ganancia educativa obtenida por los estudiantes después de haber cursado la EMS, por medio de una escala común para las pruebas ENLACE tanto de cierre de trayecto escolar de secundaria, como de bachillerato, para dar progresión a los esfuerzos de evaluación de ambos niveles.

Durante la aplicación de ENLACE-MS entre 2008 y 2010, se hicieron diversos ejercicios y experimentos para consolidar paulatinamente el diseño que se utilizaría en el estudio de ganancia educativa para los años posteriores.

A partir de 2011, la prueba ENLACE-MS se sometió a algunas modificaciones para brindar un diagnóstico acerca del grado de avance de los estudiantes en la implementación del Marco Curricular Común en la EMS. Por ello, la nueva estructura de la prueba mide indicios de competencias básicas asociadas a dos de los cuatro campos disciplinares establecidos en 
dicho Marco Curricular Común. ${ }^{4}$ De esta manera, la evaluación del área de Comunicación se adaptó para corresponder con el enfoque pedagógico tanto del campo de Comunicación (comprensión lectora) como del campo disciplinar de Matemáticas.

Posteriormente, a partir de 2015, el examen se incorporó al Plan Nacional para la Evaluación de los Aprendizajes (PLANEA) del Instituto Nacional para la Evaluación de la Educación (INEE), conservando las fortalezas conceptuales y operacionales de ENLACE-MS y transformándose en PLANEA-MS con un nuevo modelo de análisis y estimación, una nueva escala de calificación y nuevos niveles numéricos de dominio para clasificar a los sustentantes.

ENLACE/PLANEA-MS siguió una estrategia de recolección de datos que contempló la elaboración y aplicación anual de diferentes instrumentos de medición y la implementación de un diseño muestral de alumnos a los que se aplicaron los instrumentos que, en conjunto, permiten obtener los parámetros estadísticos para realizar el proceso de calificación y otros procesos con fines de investigación. Estos instrumentos fueron:

- Prueba Operativa: se aplicó de manera censal a los alumnos del último año de bachillerato y sus reactivos cada año se hacían públicos después de la aplicación. Con ella se generó información para cada alumno acerca de su nivel de desempeño en las áreas evaluadas.

- Versión Pretest: es un instrumento que se ensambla de manera matricial $^{5}$ y se aplicaba a una muestra representativa de los alumnos que respondían la prueba operativa. Se utilizó para equiparar las pruebas ENLACE/PLANEA MS cada año. La aplicación de esta prueba sirve para el piloteo de reactivos y el ensamble de la siguiente prueba operativa. De manera adicional, se utiliza para estudios especiales.

- Pruebas para el proceso de equiparación: son instrumentos de ensamble matricial y se aplicaron de forma contrabalanceada ${ }^{6}$ con el Pretest. Estas pruebas también denominadas Vinculo se utilizaron para colocar en la misma escala la prueba operativa año con año.

\footnotetext{
4 La estructura del Marco Curricular Común de le EMS se basa en los Acuerdos 442 y 444 , publicados en el Diario Oficial el 26 de septiembre y el 21 de octubre de 2008, respectivamente.

El diseño matricial implica dividir los reactivos que integran la prueba en bloques pequeños que se ensamblan para conformar cuadernillos o formas. De esta manera los alumnos solo contestan una parte de los reactivos que componen la prueba y se evita que los resultados sean afectados por la fatiga.

6 El contrabalanceo es una técnica de aplicación que consiste en suministrar los distintos cuadernillos a los alumnos con la finalidad de controlar el efecto que pudiese generar el orden de presentación de las diferentes pruebas.
} 
- Pruebas para el estudio de ganancia educativa: se ensamblaron de forma matricial y se aplicaron de forma contrabalanceada con el Pretest. Se aplicaron tanto a los alumnos de tercero de secundaria como de tercer grado de bachillerato, y se utilizaron como ancla para equiparar la prueba de ingreso (ENLACE) y egreso (ENLACE/PLANEA MS) con el propósito de obtener una medida que representara la ganancia que resulta de haber cursado la educación media superior. Estas pruebas incluyeron reactivos sobre dos asignaturas o áreas de evaluación: Comunicación y Matemáticas. Los reactivos incluidos en estas pruebas evalúan contenidos tanto de secundaria como de bachillerato. Con el fin de cumplir con los objetivos de tener una escala común entre los dos niveles educativos y para realizar el estudio de ganancia educativa, se retomaron los parámetros de los reactivos y las puntuaciones de los alumnos en las áreas de Comunicación y Matemáticas de la prueba ENLACE tercero de Secundaria.

- Cuestionarios de contexto: se administraron a muestras de alumnos y docentes. Los directivos de las escuelas que participaron en la aplicación de la prueba ENLACE/PLANEA-MS también respondían a un cuestionario. Su propósito es contar con información relacionada con variables de contexto que permitan analizar y contextualizar los resultados de los aprendizajes.

Tanto las pruebas de equiparación como las de contexto se aplicaron, año con año, entre 2009 y 2015 , a una muestra de alumnos del último ciclo de bachillerato inscritos en aquellos planteles que manifestaron interés en participar.

Todos los instrumentos se desarrollaron con base en la Metodología del Centro Nacional de Evaluación para la Educación Superior (CENEVAL), la cual sistematizó los procesos, las normas y estándares de calidad a los que debían ajustarse los planteles. El modelo consta de diez fases o etapas: diseño de evaluación, delimitación del objeto de medida, construcción del banco de reactivos, verificación cuantitativa, ensamble, aplicación, calificación, emisión de resultados, mantenimiento del examen y elaboración de material complementario. En cada una participaron especialistas organizados en cuerpos colegiados denominados comités académicos. Un Consejo Técnico se encargó de guiar, revisar y autorizar todas las decisiones que afectan a estos instrumentos y su aplicación.

El diseño muestral que se utiliza en la aplicación de ENLACE/PLANEA-MS permitió contar con los datos de un subconjunto representativo de la población a la que se aplicó las pruebas. La aplicación de la prueba 
ENLACE fue de carácter censal en cada año y se usaron muestras para los procesos de equiparación y para recabar los datos de contexto de los estudiantes. Los datos de la muestra se utilizaron para realizar la indagación sobre las variables de contexto, llevar a cabo los análisis estadísticos necesarios en la equiparación de las pruebas de un año a otro y concretar el estudio de ganancia educativa.

Cada año se realizó la selección de escuelas y de alumnos que conforman la muestra de acuerdo con los planteles que solicitan participar en la evaluación, razón por la cual el tamaño de la muestra varía en cada aplicación. El diseño muestral y las posteriores interpretaciones de los resultados consideran diferentes niveles de desagregación para los que se reportaron los resultados de ENLACE/PLANEA-MS, como el tipo de bachillerato, tipo de sostenimiento o entidad federativa.

\section{¿Cómo se analiza la ganancia educativa?}

Para realizar el estudio de ganancia educativa, se buscó que las pruebas ENLACE de secundaria y ENLACE/PLANEA MS midieran constructos ${ }^{7}$ semejantes. Se optó por tomar como medida de ingreso los resultados de ENLACE de tercero de secundaria y para la medida de egreso los correspondientes a ENLACE/PLANEA MS. Con el fin de establecer una escala común entre estos instrumentos, el proceso se apoyó con el diseño de pruebas ancla que primero, como parte de ejercicios previos, y después como instrumento consolidado se aplicaron de forma controlada y se distribuyeron de manera matricial a una muestra representativa de estudiantes, tanto de tercero de secundaria como del último ciclo de bachillerato (Esquema 1).

En 2008 se aplicó la primera prueba y se denominó Alfa. Esta prueba estaba estructurada considerando los contenidos de la prueba ENLACE tercero de Secundaria. En 2009, derivado de la Reforma Integral de la Educación Básica, se modificó la prueba Alfa y se incorporó al diseño una prueba denominada Gamma, que mantenía la estructura de ENLACE MS. En 2010 se conformó una sola prueba integrada por reactivos de las pruebas Alfa y Gamma que únicamente evaluaban contenidos comunes para secundaria y bachillerato; dicha prueba se denominó Delta. ${ }^{8}$ Ese año quedó consolidada la prueba para realizar la equiparación en el estudio de

\footnotetext{
7 Usualmente un constructo o variable latente se define como una entidad abstracta y teórica que se infiere a partir de un conjunto de conductas observables, como por ejemplo, la inteligencia, la habilidad matemática, etcétera.

8 La prueba Delta, desde su consolidación, ha evaluado a los alumnos con reactivos que permanecen iguales entre la prueba aplicada en secundaria y la que se aplica en bachillerato.
} 
ganancia educativa; su aplicación continuó en 2011, después de realizar un ajuste de contenido a una fracción de sus reactivos.

Esquema 1: Diseño de recolección de datos de las pruebas ENLACE $3^{\circ}$ de Secundaria y ENLACE/PLANEA-MS

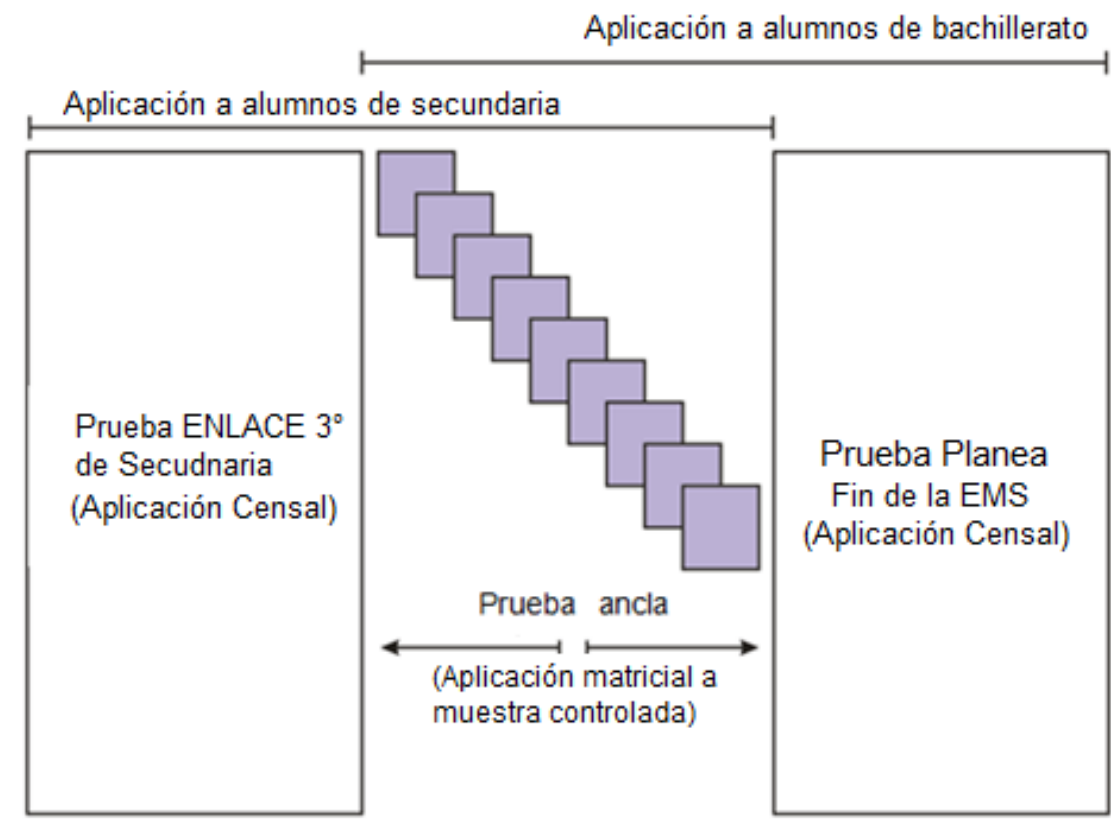

Fuente: elaboración propia con base en datos de pruebas Enlace $3^{\circ}$ secundaria y Enlace/Planea $3^{\circ}$ de educación media superior para estudio de ganancia educativa.

En 2012 se realizó, de manera preliminar y meramente experimental, la primera comparación de desempeño entre una misma generación de estudiantes (2009-2012), con el objetivo de establecer el mejor método de equiparación entre las pruebas. Lo anterior permitió que, en 2013, después de probar la eficacia de la prueba ancla y de identificar el método de transformación lineal media-sigma como el más adecuado para equiparar las pruebas de secundaria y media superior, se realizara el primer estudio formal para conocer la medida de ganancia educativa que obtuvieron los alumnos de la cohorte 2010-2013 en las áreas de Comprensión Lectora y Matemáticas tras cursar la EMS. Los siguientes dos años se continuó la 
realización del estudio para los alumnos de las generaciones 2011-2014 y 2012-2015. ${ }^{9}$

La población objeto de estudio fue la generación de alumnos de los centros de EMS de la República Mexicana que inició sus cursos de bachillerato en cada una de las tres generaciones consideradas, restringiéndose el análisis a aquellos alumnos de los que se cuenta con sus resultados en las pruebas ENLACE de tercero de Secundaria y ENLACE/PLANEA-MS. Otro criterio de inclusión al estudio fue que los alumnos hubieran respondido al menos 50 por ciento de los reactivos que integran cada una de las áreas de las pruebas.

La cantidad de alumnos contemplados para realizar el estudio de ganancia educativa en cada generación se presenta en la Tabla 1.

Tabla 1: Total de estudiantes que aplicaron la prueba ENLACE/PLANEA y los que se consideran en el estudio

\begin{tabular}{lrrrr}
\hline Generación & \multicolumn{2}{c}{ Comunicación } & \multicolumn{2}{c}{ Matemáticas } \\
\hline & \multicolumn{1}{c}{ Total } & En el estudio & Total & En el estudio \\
\hline $2010-2013$ & 994,882 & 633,142 & $1^{\prime} 002,734$ & 638,256 \\
$2011-2014$ & $1^{\prime} 004,747$ & 670,906 & $1^{\prime} 017,352$ & 679,055 \\
$2012-2015$ & $1^{\prime} 016,375$ & 657,062 & $1^{\prime} 027,016$ & 665,315 \\
\hline
\end{tabular}

Fuente: elaboración propia con base en datos de pruebas Enlace tercero de Secundaria y Enlace/ Planea tercero de educación media superior para estudio de ganancia educativa.

En congruencia con las necesidades y características del proyecto de evaluación nacional, el análisis de reactivos, la estimación de habilidad y el procedimiento de equiparación de los instrumentos se trabajaron con base en la Teoría de Respuesta al Ítem (TRI). Para el estudio de ganancia educativa, los parámetros que se obtienen a partir de los análisis estadísticos son:

- Discriminación (a). Es proporcional a la pendiente de la Curva Característica del Ítem (CCI) en el punto de inflexión. Puede interpretarse como la cualidad que tiene el reactivo de diferenciar a los sustentantes cuya habilidad está por encima o por debajo de la dificultad del reactivo. Cuanto mayor es el valor de (a), mayor será la diferencia en la probabilidad de responder correctamente al reactivo de aquellos sus-

\footnotetext{
9 La longitud de la prueba ENLACE-tercero de Secundaria fue de 57 a 67 reactivos para Comunicación, de 60 a 62 para Matemáticas; mientras que ENLACE/PLANEA-MS fue de 46 a 50 para Comunicación y 60 para Matemáticas.
} 
tentantes que se encuentran por debajo y por encima del punto (b) (la dificultad del reactivo).

- Dificultad (b). Es el valor de la variable ( $\theta$ ) sobre el que la CCI tiene su punto de inflexión. Se considera como un parámetro de posición del reactivo. Cuanto mayor sea el valor de (b), mayor será la habilidad necesaria para que la probabilidad de responder correctamente al reactivo sea superior a la probabilidad de responderlo incorrectamente.

- Pseudo-adivinación (c). Es la asíntota inferior de la CCI. Determina la probabilidad de responder correctamente al reactivo cuando la habilidad $(\theta)$ tiende a menos infinito. Puede interpretarse como la probabilidad de que el reactivo sea contestado correctamente por un alumno cuya habilidad latente tiende a menos infinito. No es estrictamente la probabilidad de una respuesta al azar, puesto que es posible que un distractor bien construido atraiga a los alumnos con menor habilidad latente, y por tano su probabilidad de responder correctamente pude ser menor que si respondiese al azar.

- Habilidad $(\theta)$. Se considera como la capacidad latente que se desea medir. Se asume que tiene una estructura cuantitativa, y aunque no directamente observable, es la causa que explica las diferencias en las respuestas a los items de la prueba. Mientras tanto, $(\theta)$ y (b) son valores de una misma escala, por lo que son directamente comparables. Por eso no se habla de una dificultad absoluta del reactivo, sino que esta dificultad viene dada por la diferencia entre $(\theta)$ y (b), es decir, $(\theta-b)$. Si esta diferencia es positiva, el reactivo es relativamente fácil para el sujeto. Si es negativa, el reactivo es relativamente difícil para el mismo.

La estimación de los parámetros y de la habilidad de los alumnos se llevó cabo mediante el programa BILOG-MG 3.0. ${ }^{10}$

Para igualar las puntuaciones entre las pruebas de ingreso y egreso (ENLACE tercero de Secundaria y ENLACE/PLANEA MS) se utilizó el método de transformación lineal media-sigma (Fórmula 1) que implicó ajustar la escala cambiando la media y la desviación estándar de las puntuaciones, pero manteniendo la distribución (Hambleton et al., 1991).

$$
\theta_{b \rightarrow s}=\theta_{b} m+n
$$

10 Bilog-MG 3.0 es un software especializado para el análisis de reactivos dicotómicos. El programa arroja resultados con base en la Teoría Clásica (índice de dificultad e índice de discriminación) y estimadores de los parámetros de la Teoría de Respuesta al Ítem (discriminación (a), dificultad (b) y pseudoazar (c)) de acuerdo con el modelo seleccionado (1, 2 ó 3 parámetros), así como funciones de información, error de estimación y estimadores de habilidad (q). 
Las constantes $m$ y $n$ se obtienen mediante:

$$
\begin{aligned}
& m=\frac{\sigma_{s}}{\sigma_{b}} \\
& n=\overline{b_{s}}-\bar{b}_{b} m
\end{aligned}
$$

Donde:

$\theta_{\mathrm{b} \rightarrow \mathrm{s}}=$ Habilidad de los alumnos de bachillerato transformada a la escala de secundaria.

$\theta_{\mathrm{b}}=$ Habilidad de los alumnos de bachillerato estimada de manera libre.

$\sigma_{\mathrm{s}}=$ Desviación estándar de los reactivos de Delta (aplicados en secundaria) que son iguales a Delta EMS.

$\sigma_{\mathrm{b}}=$ Desviación estándar de los reactivos de Delta (aplicados en bachillerato) que son iguales a Delta secundaria.

$\mathrm{b}_{\mathrm{s}}^{-}=$Media de dificultad (b) de los reactivos de Delta(aplicados en secundaria) que son iguales a Delta EMS.

$\mathrm{b}_{\mathrm{b}}^{-}=$Media de dificultad (b) de reactivos de Delta (aplicados en bachillerato) que son iguales a Delta secundaria.

Las tareas de igualación o equiparación de las puntuaciones se enfocaron, en primer lugar, en las constantes de transformación lineal y, en segundo lugar, en las puntuaciones de los alumnos para colocarlas en la misma escala.

El incremento o decremento que se observó al comparar las medias del año de terminación de secundaria y de terminación de EMS representa, para este estudio, la medida de ganancia educativa. Finalmente, se realizaron cálculos para obtener la ganancia absoluta que representa haber cursado el bachillerato, a través de la diferencia entre los resultados de la medida al ingreso (ENLACE tercero de Secundaria) y los resultados de la medida al egreso (ENLACE/Planea MS). En otras palabras y de acuerdo con Castro-Morera (2009), es la distancia que hay entre el nivel final de conocimientos y el nivel inicial (Thum, 2003), y se puede calcular a partir de la siguiente expresión:

$$
\mathrm{G}_{\mathrm{i}}=\mathrm{Y}_{\mathrm{i} 1}-\mathrm{Y}_{\mathrm{i} 0}
$$


Donde: $G_{i}$ es la ganancia educativa que obtuvo el alumno ${ }_{\mathrm{i}}$ por cursar la EMS

$Y_{i l}$ es el nivel actual de conocimientos del alumno $i$

$Y_{i 0}$ es el nivel inicial de conocimientos del alumno $i$

\section{Resultados}

Los resultados de este estudio permiten conocer sí la ganancia educativa de los subsistemas de educación media superior en México se mantuvo a través del tiempo y el paso de las generaciones. A continuación, se presenta esta medida considerando diversas características de los planteles y algunos atributos de los jóvenes que cursan la EMS.

\section{Ganancia educativa en tres generaciones de EMS}

En las figuras 1 y 2 se presentan las tendencias de las medias de habilidad al ingreso y al egreso del nivel medio superior, a nivel nacional, para las tres generaciones que participaron en los estudios de ganancia educativa.

Figura 1: Área de Comunicación. Comparativo de las medias de habilidad en los dos momentos de evaluación para las tres generaciones a nivel nacional

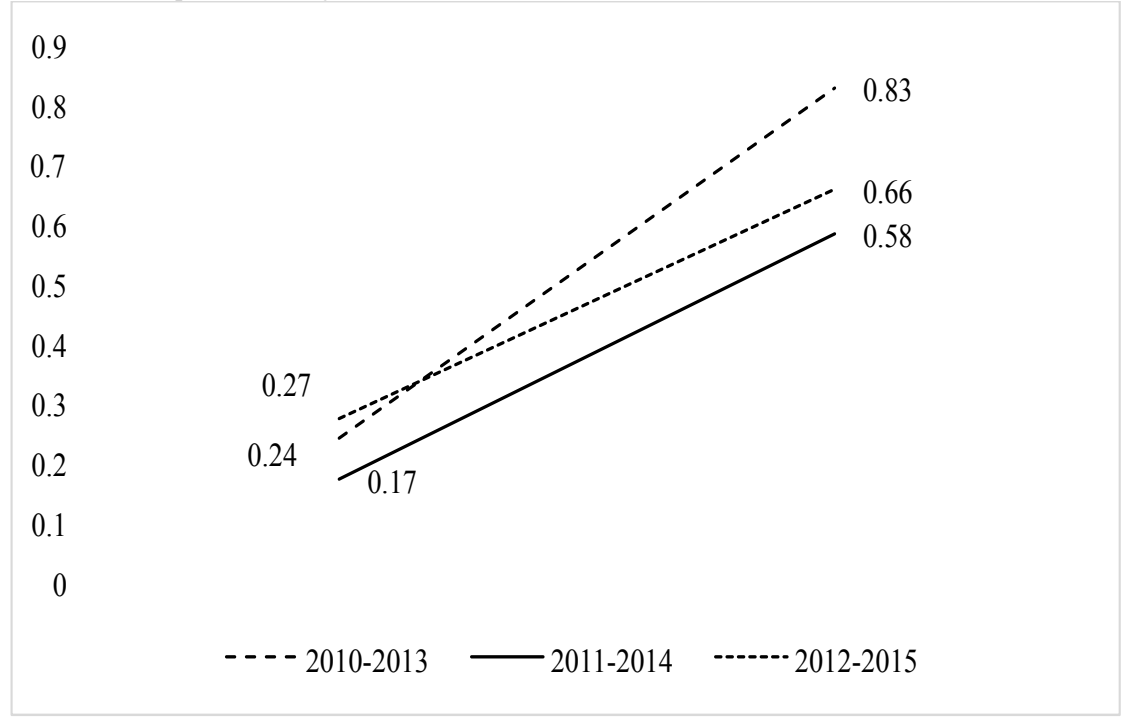

Fuente: elaboración propia con base en datos de pruebas Enlace tercero de secundaria y Enlace/Planea tercero de educación media superior para estudio de ganancia educativa. 
Figura 2: Área de Matemáticas. Comparativo de las medias de habilidad en los dos momentos de evaluación para las tres generaciones a nivel nacional

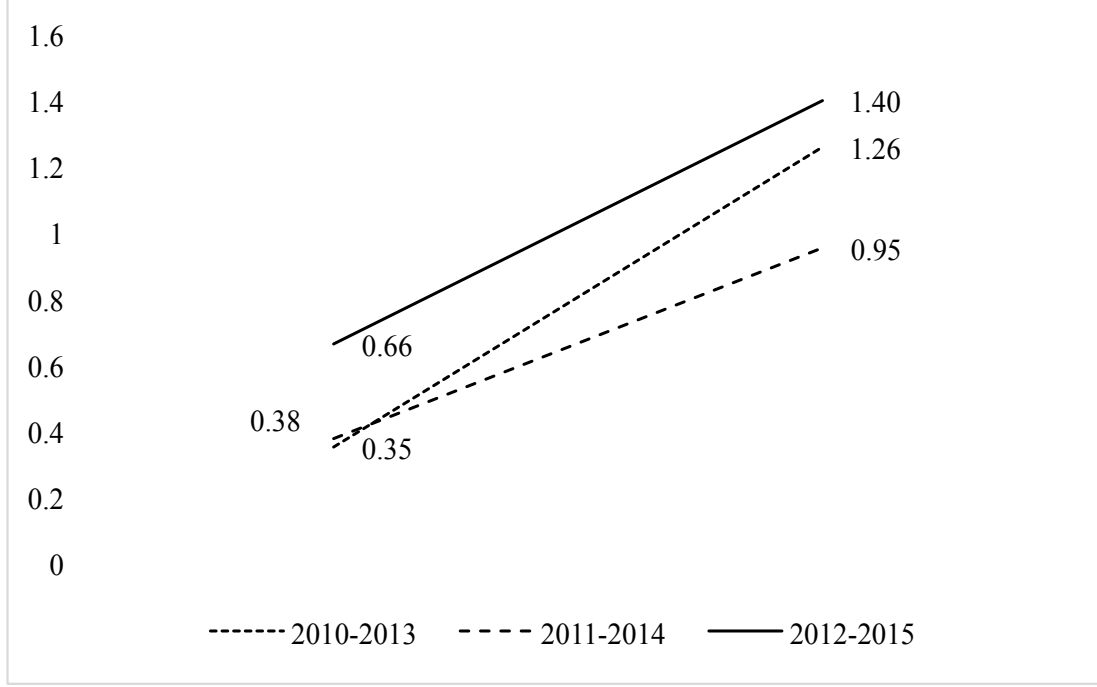

Fuente: elaboración propia con base en datos de pruebas Enlace tercero de secundaria y Enlace/Planea tercero de educación media superior para estudio de ganancia educativa.

El resultado principal que hay que resaltar es que la media de habilidad a nivel nacional experimentó un incremento, hecho que representó una ganancia educativa por haber cursado el nivel medio superior, sin importar la generación a la que hayan pertenecido en las dos áreas de conocimiento evaluadas.

Al comparar gráficamente las medias a nivel nacional, se observa que en el área de Comunicación (Figura 1), los alumnos de las tres generaciones tenían, al momento de ingresar a la educación media superior, un nivel de habilidad similar. Sin embargo, se aprecian diferencias en la distancia entre las medias de habilidad al egresar del tramo educativo, siendo más pronunciada la de la generación 2010-2013. En el área de Matemáticas (Figura 2), los alumnos de las generaciones 2010-2013 y 2011-2014 obtienen una media de habilidad al ingreso muy similar y se separan al egreso, siendo mayor la distancia entre los puntos al final para la generación 20102013. Las medias de habilidad de la generación 2012-2015 se encuentra por encima de las otras dos cohortes en ambos momentos de evaluación.

Una vez que se calculó la medida de ganancia, el análisis de varianza (ANOVA) de las medias de ganancia absoluta a nivel nacional permite demostrar que existe una diferencia estadísticamente significativa $(\mathrm{p}<0.05)$ entre las tres generaciones en ambas áreas de la prueba (Tabla 2). 


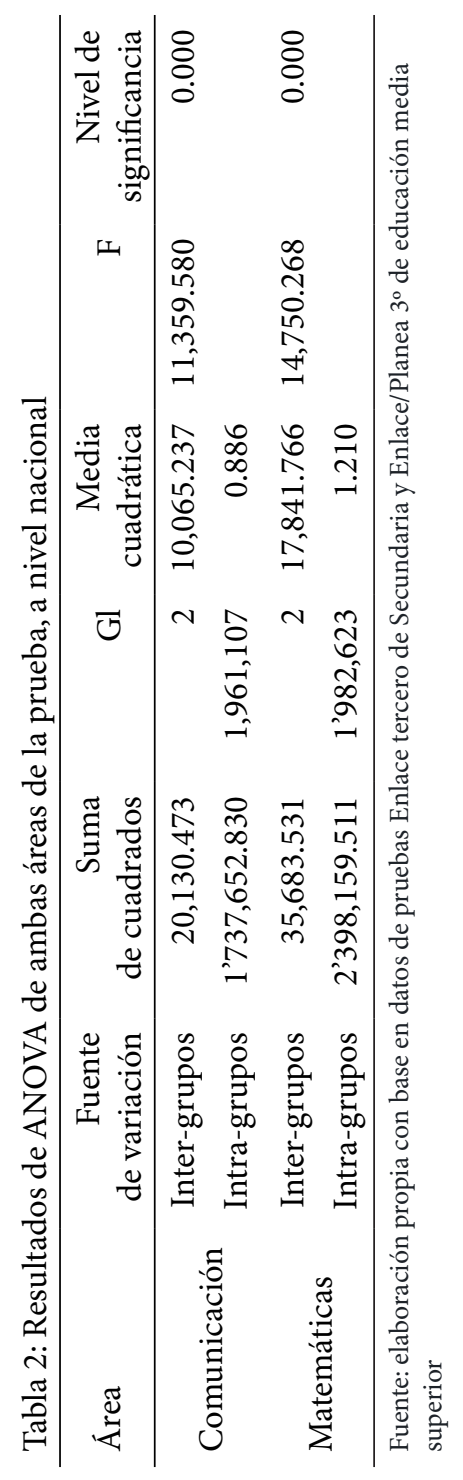


Ganancia educativa en la educación media superior / R. TUIRÁN et al.

Con la prueba T2 de Tamhane se encontró que las medias de ganancia de las tres generaciones son estadísticamente diferentes entre sí (Tabla 3) en ambas áreas de la prueba.

Tabla 3: Resultados de los comparativos múltiples de la prueba T2 Tamhane, a nivel nacional

\begin{tabular}{|c|c|c|c|c|}
\hline Área & $\begin{array}{l}\text { (I) generación } \\
\text { comparada }\end{array}$ & $\begin{array}{l}\text { (J) generación } \\
\text { comparativa }\end{array}$ & $\begin{array}{c}\text { Diferencia de } \\
\text { medias (I-J) }\end{array}$ & $\begin{array}{c}\text { Sig. } \\
\text { (valor } p \text { ) }\end{array}$ \\
\hline \multirow{6}{*}{ Comunicación } & \multirow{2}{*}{$2010-2013$} & 2011-2014 & $0.2432 *$ & 0.000 \\
\hline & & $2012-2015$ & $0.1695^{*}$ & 0.000 \\
\hline & \multirow{2}{*}{ 2011-2014 } & $2010-2013$ & $-0.2432 *$ & 0.000 \\
\hline & & $2012-2015$ & $-0.0738^{*}$ & 0.000 \\
\hline & \multirow{2}{*}{$2012-2015$} & 2010-2013 & $-0.1695^{*}$ & 0.000 \\
\hline & & $2011-2014$ & $0.0738^{*}$ & 0.000 \\
\hline \multirow{6}{*}{ Matemáticas } & \multirow{2}{*}{$2010-2013$} & 2011-2014 & $0.3293 *$ & 0.000 \\
\hline & & $2012-2015$ & $0.1716^{*}$ & 0.000 \\
\hline & \multirow{2}{*}{ 2011-2014 } & $2010-2013$ & $-0.3293 *$ & 0.000 \\
\hline & & $2012-2015$ & $-0.1578^{*}$ & 0.000 \\
\hline & \multirow{2}{*}{$2012-2015$} & $2010-2013$ & $-0.1716^{*}$ & 0.000 \\
\hline & & 2011-2014 & $0.1578^{*}$ & 0.000 \\
\hline
\end{tabular}

* La diferencia de medias es significativa al nivel 0.05 .

Fuente: elaboración propia con base en datos de pruebas Enlace $3^{\circ}$ secundaria y Enlace/Planea $3^{\circ}$ de educación media superior para estudio de ganancia educativa.

\section{Ganancia educativa en alumnos de distintos subsistemas de EMS}

En este nivel educativo existe una gran diversidad de arreglos institucionales que ofrecen servicios educativos a los jóvenes en los grados 10 a 12 de su educación. Por eso, a continuación, en la Tabla 4 se presentan los resultados de ganancia educativa para distintos subsistemas de la EMS en las tres generaciones consideradas.

En general, los alumnos de bachillerato de todos los subsistemas analizados obtienen una ganancia educativa por haber cursado este nivel, sin importar la generación a la que hayan pertenecido o el área de conocimiento evaluada. 
Tabla 4: Comparativo de las medias de habilidad en los dos momentos de evaluación para las tres generaciones de Bachillerato

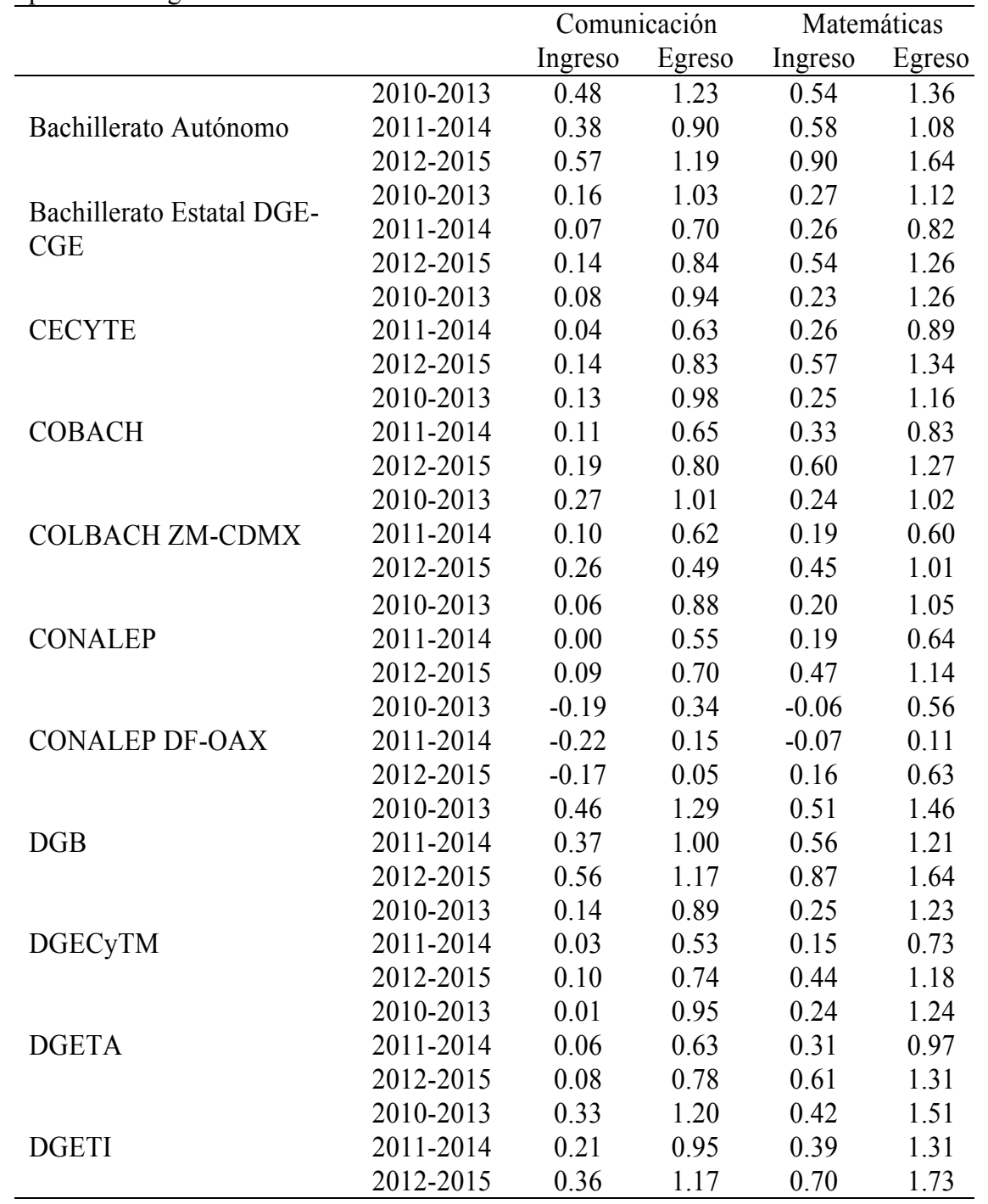

Fuente: elaboración propia con base en datos de pruebas Enlace tercero de secundaria y Enlace/Planea tercero de educación media superior para estudio de ganancia educativa.

Al comparar las medias de habilidad, se tiene que en el área de Comunicación, los alumnos de las tres generaciones tenían un nivel de habilidad semejante en los diferentes subsistemas al momento de ingresar a la educación media superior. Por supuesto, también se advierten diferencias en los 
niveles de habilidad al terminar la secundaria de los alumnos que cursaron la EMS en los distintos subsistemas.

Se registran diferencias en la distancia entre las medias de habilidad al egresar del trayecto educativo, siendo ligeramente la más pronunciada la de la generación 2010-2013. Asimismo, las medias de habilidad en Comunicación de la generación 2011-2014, tanto al ingreso como al egreso, se ubicaron por debajo de las otras dos generaciones. ${ }^{11}$

En el área de Matemáticas, los alumnos de las generaciones 2010-2013 y 2011-2014 obtienen una media de habilidad similar al ingreso y se separan al egreso de la EMS, siendo mayor la distancia entre los puntos al final para la generación 2010-2013. En tanto, las medias de habilidad de la generación 2012-2015 se encuentra por encima de las otras dos cohortes en ambos momentos de evaluación. Como se advirtió en el caso de Comunicación, también hay marcadas diferencias en las medias de logros de estos jóvenes al terminar su secundaria. ${ }^{12}$

\section{Cambio en el porcentaje de estudiantes por debajo de la media de logro de aprendizajes}

Adicional a lo realizado con los datos de la ganancia educativa de los alumnos, se complementó el estudio con un análisis de dos grupos: el grupo de referencia, esto es, el grupo del que se está reportando su evolución o medida de ganancia educativa, y otro que se denominó resto de la población, que son todos los alumnos que no pertenecen al grupo de referencia y que participaron en el estudio. Por ejemplo, si se analiza al subsistema DGETI, éste será el grupo de referencia, mientras que el resto de los subsistemas, en conjunto, son el grupo resto de la población. Se calcularon las medias de desempeño al ingreso y al egreso de la EMS para cada uno de los grupos; además se obtuvieron los porcentajes de alumnos del grupo de referencia que se encontraban por debajo de la media del resto de la población (Tabla 5). Estos porcentajes, que se reportan para las cohortes 20102013 y 2012-2015, permiten dar cuenta del avance en el desempeño de los alumnos por haber cursado el nivel educativo. Por ejemplo, si al comenzar el bachillerato, 50 por ciento de los alumnos de un determinado grupo de referencia estaban por debajo de la media del resto de la población, pero al finalizarlo este porcentaje se reduce a 45 por ciento, se puede suponer

\footnotetext{
11 Esta diferencia se corrobora como significante mediante análisis de varianza (ANOVA) de las medias de ganancia absoluta en los diferentes subsistemas.

${ }_{12}$ Con la prueba T2 de Tamhane, se encontró que las medias de ganancia de las tres generaciones son estadísticamente diferentes en ambas áreas de la prueba (salvo en el caso de Comunicación para los planteles de la DGB).
} 
que los años cursados en el bachillerato contribuyeron a un cambio de cinco puntos porcentuales. Algunos de los alumnos que previamente estaban rezagados, mejoraron su desempeño con relación a lo que se observa en el resto de la población en su conjunto.

Tanto los alumnos de la DGETI y de los CECYTE son los que presentan para las dos cohortes consideradas, y en las dos áreas de conocimiento, disminuciones en la proporción que se encontraba por debajo de la media respecto del resto de la población. Estos cambios son más marcados para la DGETI; la proporción de sus alumnos de la generación 2012-2015 que se encontraban por debajo del valor medio de logro del resto de la población pasó de 45.7 a 39.7 por ciento en Comunicación y de 48.5 a 36.1 por ciento en el área de Matemáticas (Tabla 5).

También los alumnos de la DGETA presentan en general este tipo de resultados, aunque en la última generación ya no se observa una reducción en la proporción de alumnos con logro por debajo de la media del resto de la población en Matemáticas.

Tanto los alumnos de los bachilleratos de las universidades públicas estatales, como de los planteles de DGB y particulares, no presentan avances en este indicador, pero debe apuntarse que se trata de alumnos que mayoritariamente (alrededor de 60 por ciento) ya se encontraban por encima del valor medio de logro de aprendizajes del resto de la población.

En la Tabla 4 se observa también que en algunos subsistemas existe cierta disminución de la proporción de alumnos con resultados por debajo de la media de logro del resto de la población en alguno de los dos periodos presentados y para una de las dos áreas disciplinarias analizadas, aunque no son sostenidos ni marcados.

\section{Análisis con otras variables}

Considerando solamente la generación 2012-2015, se abordan a continuación otros aspectos relacionados con la ganancia educativa estimada. El primero de éstos es el relativo al turno del plantel (matutino o vespertino) en que se estudió la EMS. Los resultados que se presentan en las figuras 3 y 4 muestran que, tanto para Comunicación como para Matemáticas, los turnos vespertinos concentran alumnos con menores niveles de logro que los alumnos que cursaron la EMS en el turno matutino, de manera que las brechas entre los dos grupos se sostienen.

Otro aspecto analizado en este estudio de ganancia educativa es el relativo al tipo de secundaria en la que estudiaron los alumnos. Se consideran cuatro tipos de secundarias: general pública, técnica pública, telesecundaria y secundarias particulares. 
Ganancia educativa en la educación media superior / R. TUIRÁN et al.

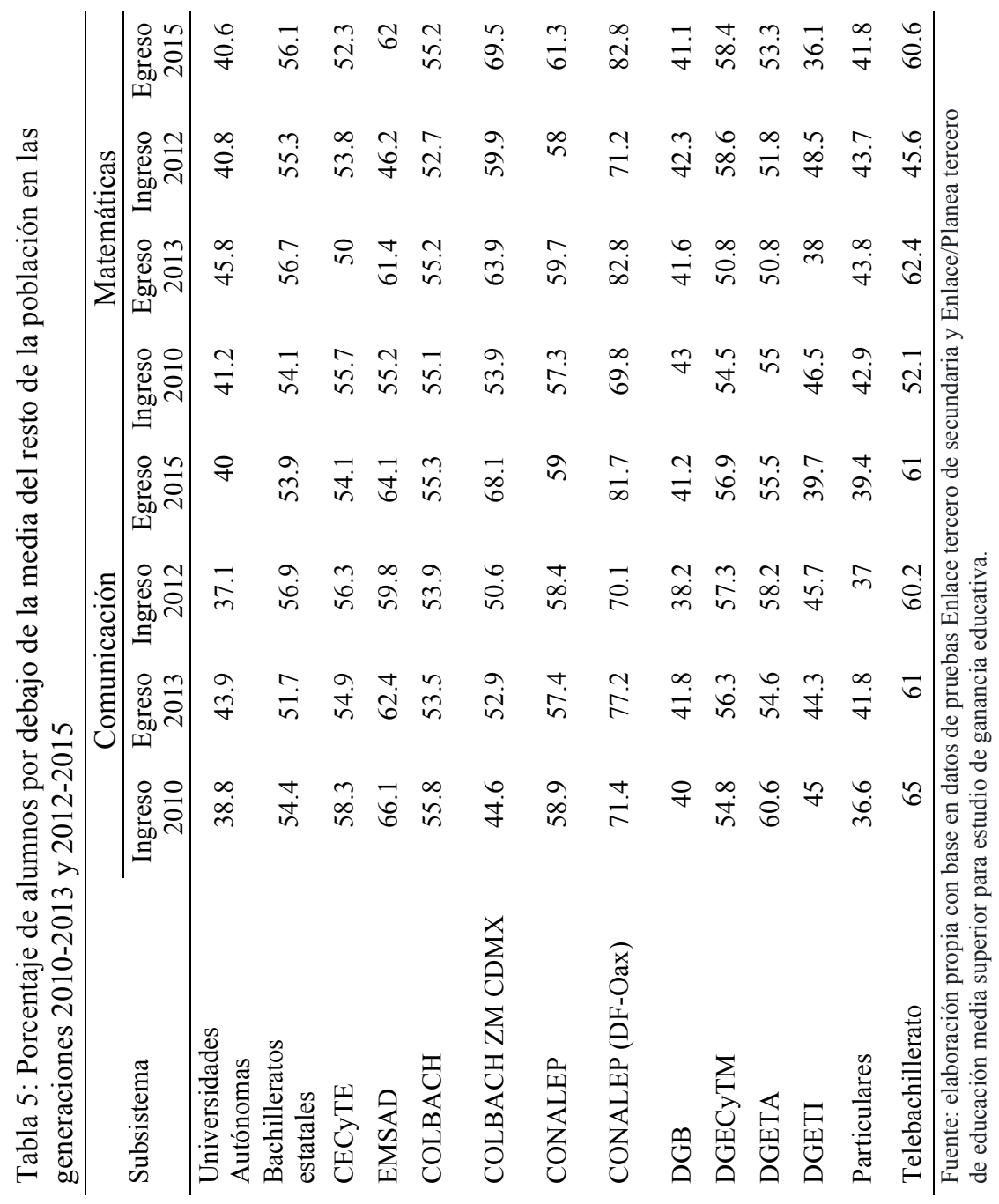


Figura 3: Área de Comunicación. Medias de logro en secundaria y media superior por tipo de turno cursado en EMS

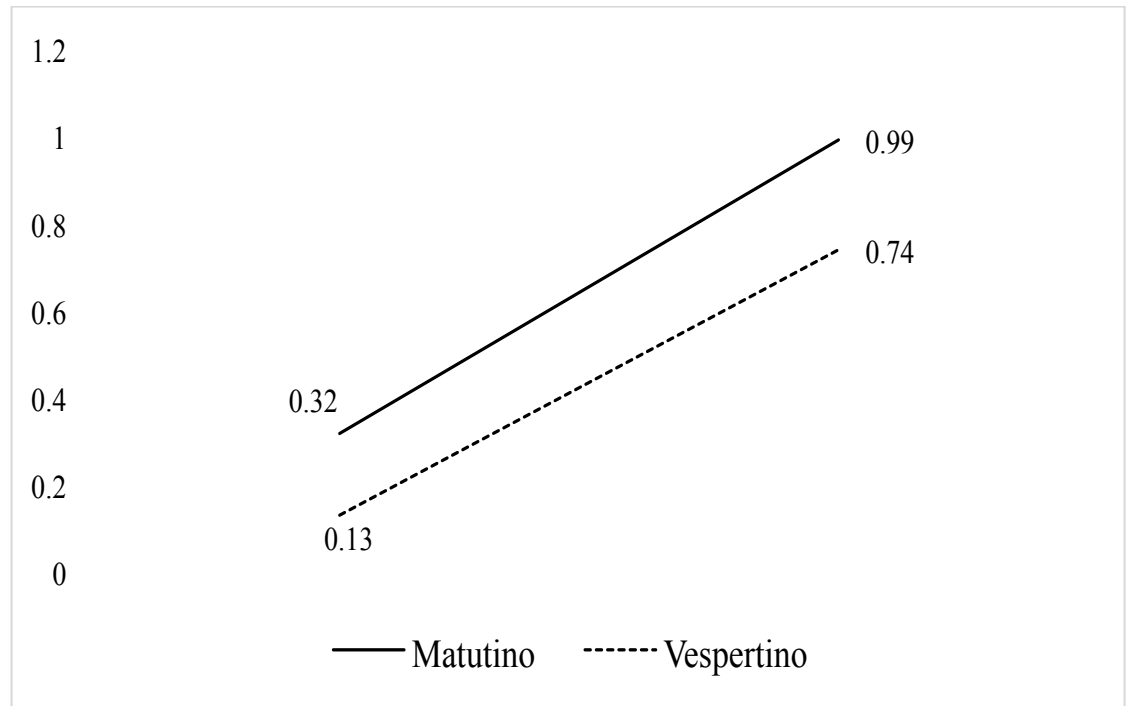

Fuente: elaboración propia con base en datos de pruebas Enlace tercero de secundaria y Enlace/Planea tercero de educación media superior para estudio de ganancia educativa.

Figura 4: Área de Matemáticas. Medias de logro en secundaria y media superior por tipo de turno cursado en EMS

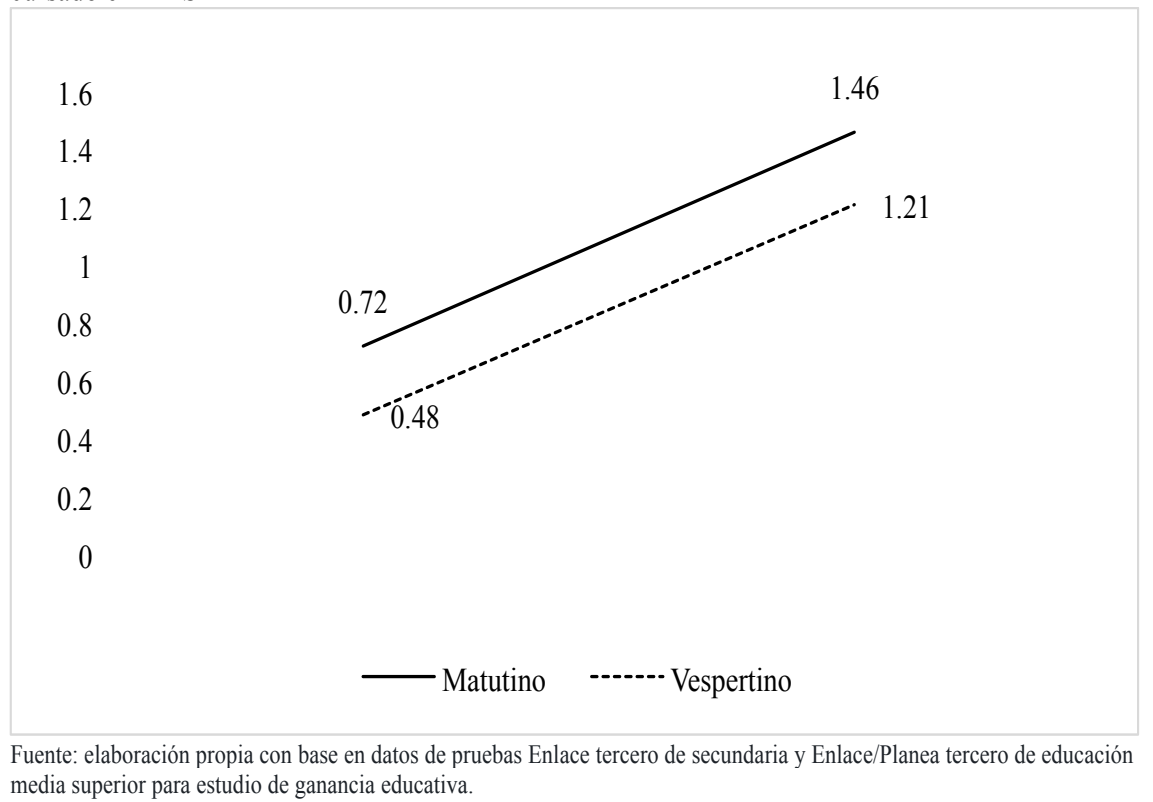


En el área de Comunicación, los alumnos de las secundarias particulares presentan una mayor ganancia educativa, aunque relativamente pequeña; en comparación a los otros tres tipos de secundarias. Entre las secundarias técnicas y las generales, los estudiantes provenientes de las primeras presentan también una ligera mayor ganancia educativa durante sus estudios de EMS. Las menores ganancia se observan en los estudiantes que cursaron la telesecundaria (Figura 5 y Tabla 6 ).

La Tabla 6 presenta las diferencias en las medias de ganancia educativa de acuerdo a la secundaria en la que estudiaron los alumnos de EMS. En cada celda se presenta la diferencia de las medias de ganancia. Si el signo es positivo, indica que la ganancia fue mayor para los jóvenes que cursaron la secundaria en la categoría del renglón respecto a la categoría en la columna.

Figura 5: Área de Comunicación. Medias de logro en secundaria y media superior por tipo de secundaria

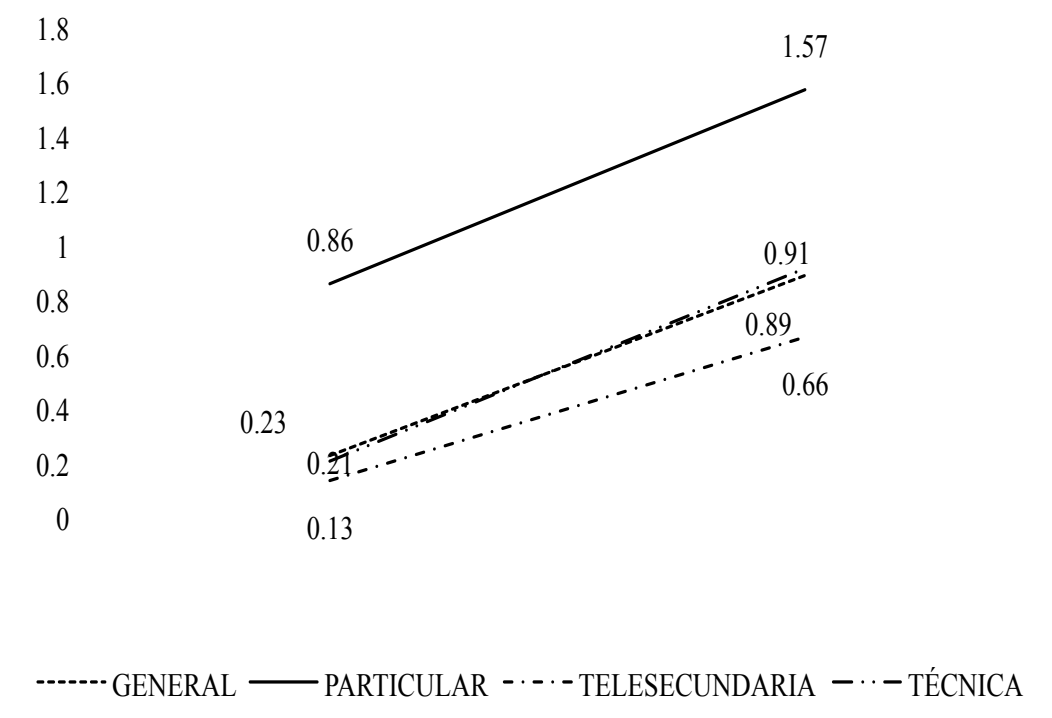

Fuente: elaboración propia con base en datos de pruebas Enlace tercero de secundaria y Enlace/Planea tercero de educación media superior para estudio de ganancia educativa.

En Matemáticas se destaca que el nivel de logro de los alumnos de secundarias técnicas y generales, que era menor que el de las telesecundarias, presentaba mayores niveles de ganancia y al concluir la EMS ya se encontraba por arriba del nivel de logro de los alumnos que provenían de las telesecundarias (Figura 6 y Tabla 6). 


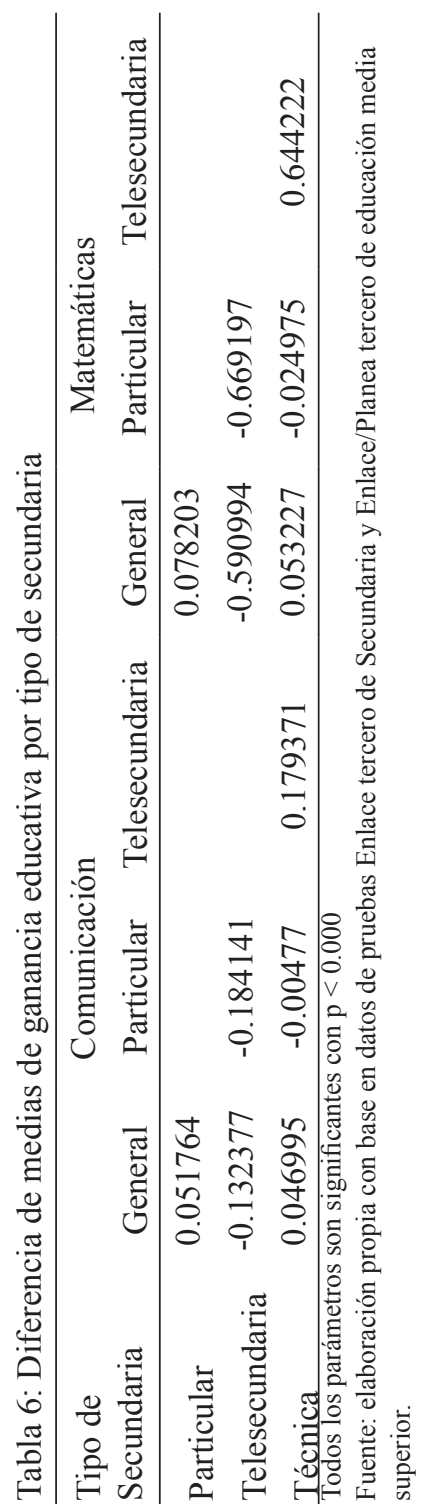


Un tercer factor analizado es el sexo de los estudiantes. Los alumnos hombres en el área de Comunicación y de Matemáticas tienden a presentar una mayor ganancia educativa que las mujeres, siendo significante estadísticamente esta diferencia (Figura 7 y Figura 8; Tabla 7). Esta situación hace que se cierre la brecha por sexo en el área de Comunicación, aunque sigue siendo mayor el nivel de logro de las mujeres.

Figura 6: Área de Matemáticas. Medias de logro en secundaria y media superior por tipo de secundaria

2.5

2

1.5

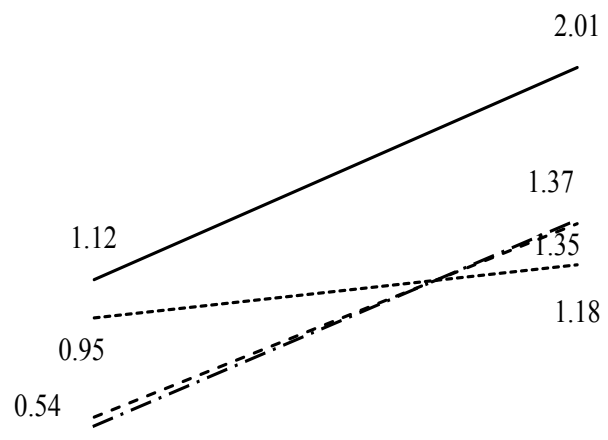

0.5

0.50

0

- - - GENERAL — PARTICULAR ---.-- TELESECUNDARIA - - TÉCNICA

Fuente: elaboración propia con base en datos de pruebas Enlace tercero de secundaria y Enlace/Planea tercero de educación media superior para estudio de ganancia educativa.

En el área de Matemáticas, se parte de niveles de logro muy similares al concluir la secundaria, pero al finalizar la EMS se amplía la brecha en favor de los hombres. ${ }^{13}$

Tabla 7: Ganancia educativa por sexo 2012-2015

\begin{tabular}{lcc}
\hline Sexo & Comunicación & Matemáticas \\
\hline Mujer & 0.6014126 & 0.811831 \\
Hombre & 0.7193645 & 0.6689919 \\
\hline
\end{tabular}

Todos los parámetros son significantes con $\mathrm{p}<0.000$

Fuente: elaboración propia con base en datos de pruebas Enlace tercero de Secundaria y Enlace/

Planea tercero de educación media superior.

13 Este resultado se analiza en el siguiente apartado. 
Figura 7: Área de Comunicación. Medias de logro en secundaria y media superior por sexo

1.2

1

0.8

0.6

0.4

0.2

0

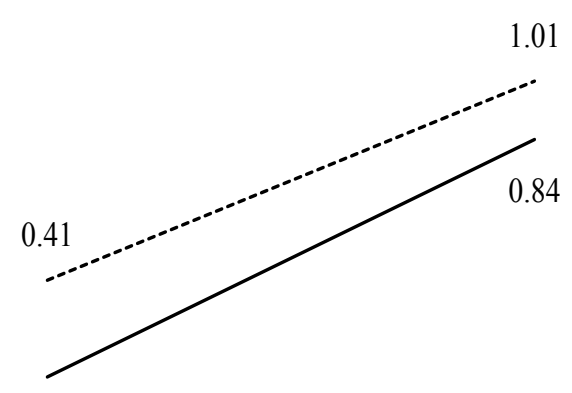

0.12

------ Mujer — Hombre

Fuente: elaboración propia con base en datos de pruebas Enlace tercero de secundaria y Enlace/Planea tercero de educación media superior para estudio de ganancia educativa.

Figura 8: Área de Matemáticas. Medias de logro en secundaria y media superior por sexo

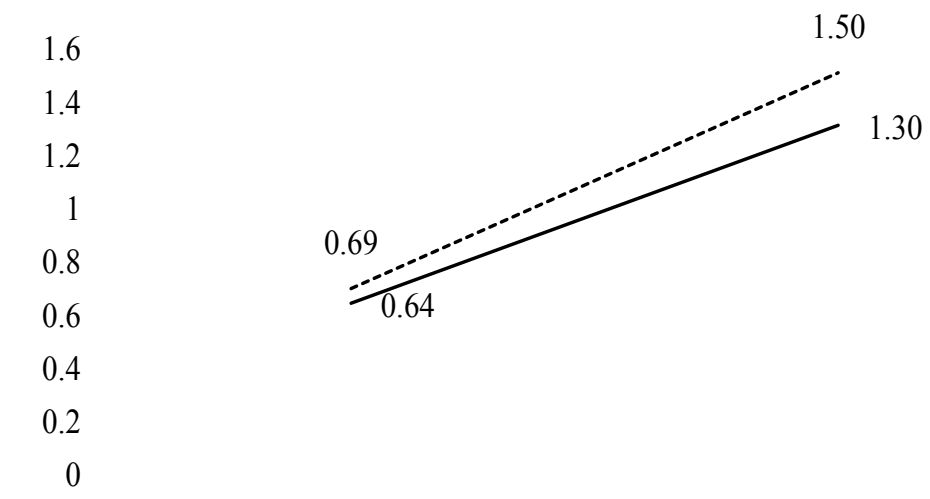

\section{- Mujer ------- Hombre}

Fuente: elaboración propia con base en datos de pruebas Enlace tercero de secundaria y Enlace/Planea tercero de educación media superior para estudio de ganancia educativa. 
Ganancia educativa en la educación media superior / R. TUIRÁN et al.

\section{Una aproximación multivariada a los elementos asociados a la ganancia educativa}

Para evaluar la relación entre las diferentes variables hasta ahora presentadas y la ganancia educativa, se realizó un análisis de regresión a través del método de mínimos cuadrados ordinarios. Los datos utilizados consideran, además de la información sobre ganancia educativa, información del Formato 911 de inicio del ciclo escolar 2015-2016 y de la prueba ENLACE 2012 aplicada a alumnos de tercero de secundaria.

Para medir el efecto de ser hombre o mujer en la variable de interés, se utilizó una variable dicotómica en la cual 1 significa hombre y 0 mujer. Con respecto al tipo de secundaria a la que asistió el alumno, se consideraron cuatro tipos: secundaria general, técnica, telesecundaria y particular. Dichas variables toman el valor de 1 cuando el alumno asistió a los tres primeros tipos de institución y 0 cuando asistió a una secundaria particular, que es la categoría de referencia para los parámetros de esta variable. Los deciles de logro se dicotomizaron para ser incluidos en el análisis. En esta variable, la categoría de referencia es el primer decil en los resultados más bajos en secundaria. Además, se incluyó en el modelo si el alumno asistió o no a un plantel de EMS perteneciente a alguna de las siguientes categorías construidas a partir de la matrícula que atiende: menos de 300 alumnos, entre 301-600 alumnos, entre 601-900 alumnos y más de 900 alumnos. Se tomó la primera categoría como referencia para las otras tres. También se consideró el tipo de sostenimiento del plantel de EMS al que asistió el alumno. Para ello, se construyeron cuatro variables categorías: autónomo, estatal, federal y particular. Los subsistemas considerados en el sostenimiento federal son DGB, DGETA, DGETI, DGECyTM, Colbach ZMCM y CONALEP Oaxaca y Ciudad de México. La categoría de planteles particulares es la de referencia. Finalmente, para estimar el efecto en la ganancia educativa de asistir al turno matutino o vespertino, se construyó una variable dicotómica que asigna el valor de 1 cuando el alumno asiste a la escuela en la mañana y 0 cuando asiste por la tarde.

Tabla 8: Estadística descriptiva de la variable ganancia

\begin{tabular}{lcccc}
\hline Área & Media & $\begin{array}{c}\text { Desviación } \\
\text { estándar }\end{array}$ & Mínimo & Máximo \\
\hline Comunicación & 0.6565494 & 0.9727417 & -5.249828 & 5.754768 \\
Matemáticas & 0.7357717 & 1.09842 & -5.635805 & 6.659812 \\
\hline
\end{tabular}

Fuente: elaboración propia con base en datos de pruebas Enlace tercero de Secundaria y Enlace/Planea tercero de educación media superior. 
En el análisis, ganancia educativa es la variable dependiente. La estadística descriptiva de la variable se presenta en la Tabla 8, tanto para Comunicación como para Matemáticas.

En la Tabla 9, los resultados de los modelos de regresión indican que:

- La ganancia educativa es mayor en los hombres que en las mujeres (parámetros positivos para la categoría respecto a las mujeres), con efectos más grandes en el área de Matemáticas.

- En el área de Comunicación, los alumnos de EMS en planteles más pequeños o con más de 900 estudiantes presentan ganancias significativamente mayores que los de planteles entre 300 y 899 alumnos. En el caso de la ganancia educativa para Matemáticas, mientras más alumnos tiene el plantel, mayor es la ganancia educativa en esta área disciplinar.

- Los alumnos de los turnos matutinos tienen mayor ganancia educativa que los de los turnos vespertinos.

\section{Alcances y limitaciones}

Los resultados aquí presentados indican una mejora en el rendimiento de los alumnos después de haber cursado la educación media superior. Éstos pueden considerarse como indicios relevantes de que los años cursados en el bachillerato ofrecen una ganancia en lo referente a la educación, independientemente de la institución, sus características y el lugar donde ésta se ubique.

Aunque se tiene un referente empírico acerca del cambio en el nivel de logro de aprendizajes de los estudiantes después de tres ciclos escolares en el bachillerato, es importante considerar que este análisis se centra únicamente en dos áreas de aprendizaje.

Además, para el cálculo del indicador de ganancia educativa sólo se contó con aproximadamente 65 por ciento de los datos de los alumnos de las generaciones que presentaron la prueba de media superior y la de ENLACE tercero de secundaria. El 35 por ciento restante corresponde a:

1. Estudiantes que resolvieron menos de 50 por ciento de alguna de las áreas de la prueba.

2. Estudiantes que respondieron solo a una de las pruebas (Secundaria o Media Superior). 
Ganancia educativa en la educación media superior / R. TUIRÁN et al.

3. Estudiantes que no contaban con la CURP completa o ésta fue incorrectamente codificada.

4. Estudiantes que abandonaron sus estudios (por diferentes razones) o repitieron grado.

Tabla 9: Parámetros de regresión de la ganancia educativa 2012-2015

\begin{tabular}{lcc}
\hline Variable & Comunicación & Matemáticas \\
\hline Sexo & $0.0233^{* * *}$ & $0.177^{* * *}$ \\
Secundaria general & $-0.290^{* * *}$ & $-0.413^{* * *}$ \\
Telesecundaria & $-0.456^{* * *}$ & $-0.727^{* * *}$ \\
Secundaria Técnica & $-0.254^{* * *}$ & $-0.379^{* * *}$ \\
Decil de logro 2 & $-0.442^{* * *}$ & $-0.391^{* * *}$ \\
Decil de logro 3 & $-0.759^{* * *}$ & $-0.738^{* * *}$ \\
Decil de logro 4 & $-1.021^{* * *}$ & $-1.030^{* * *}$ \\
Decil de logro 5 & $-1.240^{* * *}$ & $-1.285^{* * *}$ \\
Decil de logro 6 & $-1.416^{* * *}$ & $-1.557^{* * *}$ \\
Decil de logro 7 & $-1.591^{* * *}$ & $-1.858^{* * *}$ \\
Decil de logro 8 & $-1.843^{* * *}$ & $-2.183^{* * *}$ \\
Decil de logro 9 & $-2.233^{* * *}$ & $-2.546^{* * *}$ \\
Decil decil de 10 & $-2.715^{* * *}$ & $-2.996^{* * *}$ \\
De 300 a 600 alumnos & $-0.0437^{* * *}$ & $0.0489^{* * *}$ \\
De 601 a 900 alumnos & $-0.0157^{* * *}$ & $0.117^{* * *}$ \\
Más de 900 alumnos & $0.0203^{* * *}$ & $0.210^{* * *}$ \\
Autónomo & $0.187^{* * *}$ & $0.154^{* * *}$ \\
Estatal & $0.118^{* * *}$ & $0.0666^{* * *}$ \\
Federal & $0.196^{* * *}$ & $0.215^{* * *}$ \\
Turno matutino & $0.124^{* * *}$ & $0.126^{* * *}$ \\
Constante & $1.965^{* * *}$ & $2.074^{* * *}$ \\
Observaciones & 572,804 & 580,192 \\
R-cuadrada & 0.123 & 0.292 \\
\hline
\end{tabular}

*** $\mathrm{p}<0.01, * * \mathrm{p}<0.05, * \mathrm{p}<0.1$

Fuente: elaboración propia con base en datos de pruebas Enlace tercero de Secundaria y Enlace/

Planea tercero de educación media superior.

Para explorar qué tipo de efecto podría introducir esta característica, a continuación se presentan los datos sobre los niveles de logro en ENLACE/ 
PLANEA-MS, considerando los datos totales de los alumnos que presentaron la prueba y los correspondientes a los estudiantes en este estudio (para los que se pudieron vincular los datos individuales de logro en tercero de secundaria con los de tercero de EMS). En las tablas 10 y 11 se observa que la población en este estudio tiene una menor proporción de alumnos en el grupo con menor nivel de logro tanto en el área de comunicación como de matemáticas1 (Nivel I).

Tabla 10: Distribución porcentual de alumnos de EMS de acuerdo con el nivel de logro de aprendizajes: población que realizó el examen y considerada en el estudio de ganancia educativa. Área de Comunicación

\begin{tabular}{ccccccc}
\hline \multirow{2}{*}{$\begin{array}{c}\text { Nivel de } \\
\text { logro }\end{array}$} & \multicolumn{2}{c}{2015} & \multicolumn{2}{c}{2014} & \multicolumn{2}{c}{2013} \\
\cline { 2 - 7 } & PLANEA & Estudio & ENLACE & Estudio & ENLACE & Estudio \\
\hline I & 21.8 & 19.5 & 19.1 & 17.7 & 16.4 & 15.0 \\
II & 34.8 & 33.9 & 36.3 & 35.3 & 33.6 & 32.6 \\
III & 37.9 & 40.3 & 40.1 & 41.9 & 43.9 & 45.6 \\
IV & 5.5 & 6.3 & 4.6 & 5.2 & 6.1 & 6.8 \\
\hline
\end{tabular}

Fuente: elaboración propia con base en datos de pruebas Enlace tercero de Secundaria y Enlace/ Planea tercero de educación media superior.

Esta evidencia sugiere que algunos alumnos de menor rendimiento académico en secundaria probablemente no lograron transitar en esos años a la EMS o no alcanzaron avanzar hacia el tercer grado de bachillerato (que es cuando se realiza la prueba ENLACE/PLANEA), al haber reprobado o abandonado sus estudios en los dos primeros años de este nivel educativo. En consecuencia, esto podría implicar un posible efecto de sobre-estimación de la ganancia educativa.

Tabla 11: Distribución porcentual de alumnos de EMS de acuerdo con el nivel de logro de aprendizajes: población que realizó el examen y considerada en el estudio de ganancia educativa. Área de Matemáticas

\begin{tabular}{ccccccc}
\hline $\begin{array}{c}\text { Nivel de } \\
\text { logro }\end{array}$ & \multicolumn{2}{c}{2015} & \multicolumn{2}{c}{2014} & \multicolumn{2}{c}{2013} \\
PLANEA & Estudio & ENLACE & Estudio & ENLACE & Estudio \\
\hline I & 26.1 & 23.4 & 26.6 & 24.6 & 28.3 & 26.6 \\
II & 35.4 & 34.9 & 34.1 & 33.5 & 35.4 & 34.9 \\
III & 18.0 & 18.9 & 20.0 & 20.7 & 20.2 & 21.0 \\
IV & 20.5 & 22.8 & 19.4 & 21.2 & 16.1 & 17.5 \\
\hline
\end{tabular}

Fuente: elaboración propia con base en datos de pruebas Enlace tercero de Secundaria y Enlace/ Planea tercero de educación media superior. 
Ganancia educativa en la educación media superior / R. TUIRÁN et al.

\section{CONSIDERaCiones Finales}

Los resultados del análisis indican que los estudiantes presentan ganancia educativa en las áreas de Comunicación y Matemáticas con el tránsito por la EMS.

Sin embargo, este efecto no es homogéneo para todos los estudiantes de la EMS y las brechas no desaparecen por completo al concluir este nivel educativo. Existen diferencias de género en la ganancia educativa, de manera que las mujeres no sólo tienen desempeños de logro de aprendizajes más bajos en matemáticas. Esto se asocia al hecho de que tienden a ser las que presentan menores ganancias. Asimismo, los estudiantes que provienen de telesecundarias tampoco presentan altos niveles de ganancia educativa.

Es necesario indagar más acerca de qué están haciendo los subsistemas en los que más avanzan los estudiantes y cuáles son las características de los aprendizajes en esos planteles. Y también desarrollar medidas de afirmación positiva en la EMS para favorecer mayores aprendizajes para las mujeres y los estudiantes que provienen de secundarias a las que asisten adolescentes del medio rural o urbano marginado.

Este trabajo muestra también la importancia de contar con instrumentos de evaluación de los aprendizajes que cuenten con elementos comparables en el tiempo y que puedan ser vinculados entre niveles educativos a lo largo de la trayectoria de los estudiantes, con el fin de hacer posible la medición de la ganancia educativa y la exploración de sus determinantes.

\section{REFERENCIAS BIBLIOGRÁFICAS}

Value-Added Model (VAM) Research for Educational Policy: Framing the Issue, 2013, en Education Policy Analysis Archives 21, núm. 4/5: 1-11.

Castro-Morera, M., 2009, "La evaluación educativa desde la perspectiva del valor añadido", en Estudios sobre educación, 16 (147-166).

Ceneval, 2011, Metodología Ceneval. México.

Ceneval, 2015, Manual técnico ENLACE Media Superior 2013-2014. Evaluación Nacional de Logro Académico en Centros Escolares de Educación Media Superior. Recuperado de http://www.enlace.sep.gob.mx/content/ms/docs/Manual_Tecnico_ENLACE_MS_2013_2014.pdf

Cervini, R. y Dari, N., 2008, "Algunos problemas metodológicos en los estudios de eficacia escolar: una ilustración empírica”, en Eficacia escolar y factores asociados en América Latina y el Caribe. UNESCO y LLECE, Chile (49-59). 
Chudowsky, N., Koenig, J. A., Braun, H. I., 2010, Getting value out of value-added : report of a workshop. National Research Council (U.S.), and National Academy of Education, Washington: National Academies Press.

Diario Oficial de la Federación, 2008a, Acuerdo 442, Disponible en http://dof.gob. $\mathrm{mx} /$ nota_detalle.php ?codigo $=5061936 \&$ fecha $=26 / 09 / 2008 /$

Diario Oficial de la Federación, 2008b, Acuerdo 444 Disponible en http://dof.gob. $\mathrm{mx} /$ nota_detalle. php? codigo $=5064951 \& \mathrm{fecha}=21 / 10 / 2008$

Downing, S. y Haladyna, T., 2012, Manual para el desarrollo de pruebas a gran escala. México: Ceneval.

Du Toit, M., 2003, IRT from SSI: Bilog-MG, Multilog, Parscale, Testfact. Lincolnwood, IL: Scientific. Software International.

Gaviria, J.L. y Castro, M., 2005, Modelos jerárquicos lineales. La Muralla, España.

Gaviria, J.L., 2006, El valor añadido en educación y la función de producción educativa. Recuperado de http://www.adide.org/revista/index.php?option=comcontent\&task=view\&id=20\&Itemid=29

Gaviria, J.L., Biencinto, C. y Navarro, E, 2009, "Invarianza de la estructura de covarianzas de las medidas de rendimiento académico en estudios longitudinales en la transición de Educación Primaria a Secundaria”, en Revista de educación, núm. 348 (Ejemplar dedicado a: El valor añadido en educación), 153-174.

Gaviria, J.L. y Castro, M., 2010, Modelos de valor añadido en educación. Universidad Complutense, Seminario Ceneval-INEE, mayo.

Goldstein, H., 2003, Multilevel Satistical Models, London: Arnold.

Hambleton, R., Swaminathan, H., 1985, Item response theory. Principles and applications. Assinippi Park, MA, Kluwer Academic Publishers.

Hambleton, R., Swaminathan, H. y Rogers, H., 1991, Fundamentals of item response theory, Newbury Park, CA, Sage Publications.

Kane, Michael T., 2017, Measurement Error and Bias in Value-Added Models. Research Report. ETS RR-17-25. ETS Research Report Series.

Kolen, M. J., and Brennan, R. L., 2004, Test Equating, Scaling, and Linking: Methods and Practices. EU: Springer.

Kreft, I. G. G. y De Leeuw. J., 1998, Introducing multilevel modelling. Sage Publications, London.

Miranda, L., 2008, "Factores asociados al rendimiento escolar y sus implicaciones para la política educativa del Perú", en Eficacia escolar y factores asociados en América Latina y el Caribe. UNESCO y LLECE, Chile (185-207).

Simkovic, Michael, 2017, "A Value-Added Perspective on Higher Education”, en UC Irvine Law Review, núm. 1: 123. 
Snijders, T.A.B. y Bosker, R.J., 1999, Multilevel Analysis. An Introduction to basic and advanced multilevel modelling. Sage Publications, London.

Subsecretaría de Educación Media Superior, 2008, Reforma Integral de la Educación Media Superior en México: La Creación de un Sistema Nacional de Bachillerato en un marco de diversidad. México: SEP.

Subsecretaría de Educación Media Superior, 2009, Competencias Disciplinares Básicas para la Educación Media Superior. México: SEP.

Thum, Y. M., 2003, "Measuring progress towards a goal: Estimating teacher productivity using a multivariate multilevel model for value-added analysis", en Sociological Methods and Research, 32(2), 153-207.

Yeow Meng Thum, 2009, No child left behind: methodological challengs and recommendations for measuring adequate yearly progress. Michigan State University. College of Education, EUA. (67-90).

\section{RESUMEN CURRICULAR DE LOS AUTORES}

\section{Rodolfo Tuirán}

Doctor en sociología por la Universidad de Texas en Austin. Es autor o coordinador de 30 libros y más de 200 artículos en libros y revistas especializadas y de difusión en temas sociodemográficos, urbanos y educativos. En el ámbito académico, el Dr. Tuirán fue profesor-investigador del Centro de Estudios Demográficos y de Desarrollo Urbano de El Colegio de México (1983-1994) y profesor de la Facultad Latinoamericana de Ciencias Sociales (1993-1998), así como investigador invitado del Instituto Tecnológico Autónomo de México (2006). En la Administración Pública se desempeñó en el último cuarto de siglo como Secretario General del Consejo Nacional de Población (entre 1994 y marzo de 2002), Subsecretario de Desarrollo Urbano y Ordenación del Territorio de la Secretaría de Desarrollo Social (entre abril de 2002 y enero de 2006), Subsecretario de Educación Superior de la SEP (entre diciembre de 2006 a noviembre de 2012), Subsecretario de Educación Media Superior (entre diciembre de 2012 y enero de 2018), y volvió nuevamente a desempeñarse como Subsecretario de Educación Superior (de enero de 2018 a noviembre del mismo año). En 2004 recibió el Premio Nacional de Demografía; además, siete Universidades Públicas Estatales le confirieron entre 2012 y 2017 el grado de Doctor Honoris Causa; también la Universidad Autónoma de Ciudad Juárez creó en su honor la "Cátedra Patrimonial Dr. Rodolfo Tuirán". Dirección electrónica: rtuiran@colmex.mx Registro ORCID: http://orcid.org/0000-0003-2472-8948 


\section{José Luis Gaviria Soto}

En la actualidad es Catedrático de Métodos de Investigación en Educación en la Universidad Complutense de Madrid y fue director del Departamento de Métodos de Investigación y Diagnóstico en Educación de la misma universidad en el periodo 2006 a 2014. Fue presidente de la Sociedad Española de Pedagogía de septiembre de 2008 a septiembre de 2016. Es editor jefe de la Revista de Educación, publicada por el Ministerio de Educación, Cultura y Deportes del Gobierno de España. Está especializado en Evaluación de Sistemas Educativos, con especial énfasis en los problemas de medida y análisis de datos específicos de dichas evaluaciones. Ha actuado como consultor para varios gobiernos autónomos y para distintas instituciones españolas y latinoamericanas encargadas de la evaluación de sus respectivos sistemas educativos.

Dirección electrónica: josechogs@gmail.com

\section{Rubén Lugo Campos}

Tiene una licenciatura en Psicología por la Universidad Nacional Autónoma de México. Actualmente colabora como coordinador de análisis y calificación en la Dirección de Acreditación y Certificación del Conocimiento del Centro Nacional de Evaluación para la Educación Superior (Ceneval, A.C.) tiene especial énfasis en investigación educativa, análisis psicométrico de instrumentos, análisis estadístico, diseño y elaboración de instrumentos de evaluación, investigación en evaluación cualitativa e investigación del impacto educativo de las instituciones. Ha participado en proyectos de investigación así como en la elaboración de estudios del avance en el logro escolar de los alumnos de Telebachillerato Comunitario (generaciones 2013-2016, 2014-2017 y 2015-2018), Ganancia Educativa de la Educación Media Superior en el marco de la evaluación ENLACE MS, Perfiles de Ingreso de aspirantes al CONALEP, sobre la Eficacia Educativa de plantes de Profesional Técnico, Trayectoria Educativa y análisis de la capacidad diferencial de reactivos de instrumentos de evaluación.

Dirección electrónica: ruben.lugo@ceneval.edu.mx

\section{Daniel Hernández Franco}

Economista y Maestro en Salud Pública. Actualmente es investigador de la Iniciativa de Educación con Equidad y Calidad de la Escuela de Gobierno y Transformación Pública del Tecnológico de Monterrey. Fue Coordinador Nacional de Progresa y de Oportunidades, así como Coordinador de Asesores de la SEP, de SEDESOL y del CONAPO. En la Coordinación 
Sectorial de Desarrollo Académico de la Subsecretaría de Educación Media Superior dirigió la estrategia contra el abandono escolar, las acciones de formación continua docente, y la actualización del currículo de referencia de los bachilleratos tecnológicos. Impulsó el uso de los resultados de pruebas estandarizadas de logro educativo por las comunidades escolares del bachillerato para establecer procesos de mejora en el trabajo docente. Dirigió en 2018 la operación de más de 500 planteles de bachillerato tecnológico agropecuario y de ciencias del mar.

Dirección electrónica: hernandezfranco@gmail.com

Registro ORCID: http://orcid.org/0000-0002-0096-9597

\section{Miriam Benitez Isidoro}

Es maestra en Desarrollo Internacional y Política Pública por la Universidad de Chicago y licenciada en Ciencia Política y Relaciones Internacionales por el Centro de Investigación y Docencia Económicas. En la Subsecretaría de Educación Media Superior, se desempeñó como asesora de la Coordinación Sectorial de Desarrollo Académico y como Subdirectora de planeación. Tuvo a su cargo el seguimiento y análisis de los resultados de las pruebas estandarizadas de logro de los aprendizajes, así como el seguimiento y evaluación de la Estrategia Nacional de Capacitación Continua Docente y de intervenciones de política pública relacionadas con la expansión de la cobertura en la Educación Media Superior. Es parte de la primera generación del programa de becarios de la Obama Foundation.

Dirección electrónica: miriam.bntez@gmail.com

Artículo recibido el 14 de marzo de 2019 y aprobado el 7 de mayo de 2019. 